Sharif University of Technology
Scientia Iranica

\title{
Risk-based cooperative scheduling of demand response and electric vehicle aggregators
}

\author{
P. Aliasghari, B. Mohammadi-Ivatloo*, and M. Abapour \\ Department of Electrical and Computer Engineering, University of Tabriz, 29 Bahman Blvd., Tabriz, Iran.
}

Received 21 June 2019; accepted 14 September 2019

\author{
KEYWORDS \\ Demand Response \\ Aggregator (DRA); \\ Electric Vehicles \\ Aggregator (EVA); \\ Cooperative \\ framework; \\ Information-Gap \\ Decision Theory \\ (IGDT); \\ Uncertainty.
}

\begin{abstract}
This paper proposes a new cooperative scheduling framework for Demand Response Aggregators (DRAs) and Electric Vehicle Aggregators (EVAs) in a day-ahead market. The proposed model implements the Information-Gap Decision Theory (IGDT) to optimize the scheduling problem of the aggregators, which guarantees to obtain the predetermined profit by the aggregators. In the proposed model, the driving pattern of electric vehicle owners and the uncertainty of day-ahead prices are simulated via scenariobased and bi-level IGDT-based methods, respectively. The DRA provides DR from two demand-side management programs including Time-Of-Use (TOU) and reward-based DR. Then, the obtained DR is offered in day-ahead markets. Furthermore, the EVAs not only meet the EV owners' demand economically, but also participate in the day-ahead market while are willing to set DR contracts with the DRA. The objective function is to maximize the total profit of DR and EV aggregators by pursuing two different strategies to deal with price uncertainty, i.e., risk-seeking strategy and risk-averse strategy. The proposed plan is formulated in a risk-based approach and its validity is evaluated with respect to a case study with realistic data of electricity markets.
\end{abstract}

(C) 2019 Sharif University of Technology. All rights reserved.

\section{Introduction}

\subsection{Motivation}

Demand Response (DR) has been introduced as a promising strategy to deal with the growing demand of energy in the world. Various DR programs have been employed by different players in the electricity market. Market operators, for example, implement DR programs to satisfy both the demand of the electricity market and power system security issues. The necessity of expanding the generation and transmission facili-

\footnotetext{
*. Corresponding author. Tel.: +9841 33393744 ;

Fax: +984133300829

E-mail addresses: p.aliasghari@tabrizu.ac.ir (P.

Aliasghari); bmohammadi@tabrizu.ac.ir (B.

Mohammadi-Ivatloo); abapour@tabrizu.ac.ir (M. Abapour)
}

doi: $10.24200 /$ sci. 2019.53685 .3446 ties could be postponed [1] by implementing proper demand-side programs. DR programs are also beneficial for transmission network providers to operate their network in a more reliable and secure condition. In addition, retailers employ DR programs to mitigate the risk of fluctuations in electricity prices in energy markets. To obtain these advantages, DR programs should be designed such that the profits of wholesalers, retailers, and customers in electricity markets would be guaranteed to make sufficient motivation for participating in these programs [2].

New DR actions are developed by Independent System Operators (ISOs) to increase the role of demand-side resources in electricity markets. In this regard, Demand Response Aggregators (DRAs) have been introduced as an effective interface agency between end-users and wholesalers to enhance the positive results of implementing DR programs. For 
example, the Australian Energy Market Commission (AEMC) has highlighted the role of active DRAs as one of the main solutions in flourishing DR outcomes. Moreover, with the breakthrough of technology and communications industry, new consumers of electricity, such as Electrical Vehicles (EVs), have been introduced in recent decades. Since vehicles are driven only about $5 \%$ of time and remain stationary quite often [3], there can be a new target market for DRAs as flexible loads.

In recent years, the penetration of $\mathrm{EVs}$ has increased to respond to the concerns of fossil fuel reduction and environmental issues $[4,5]$. On the other hand, if the electrical load imposed by EVs could not be controlled correctly, it would bring about problems for power systems in both security and economic operation areas [6-8]. EVs owners, however, can even facilitate the operation of power systems by managing their consumption and participating in energy markets actively as an energy storage system and flexible load. It is obvious that taking part in energy markets individually could not be beneficial for EV owners in terms of time and economy due to the uncertainty of electricity prices [9]. Therefore, an interface agency, called EV Aggregator (EVA), must be introduced to enhance the advantages of charging and discharging of thousands of EVs in comparison with individual EVs [10]. As a matter of fact, EVA can play a significant role in mediating between power system networks and EV owners and participate as a representative of vehicle owners in energy markets [10]. As a result, the optimal scheduling of EVAs can bring about profit for EV owners, power system operators, and EVAs.

Both DRAs and EVAs as intermediate agencies have faced two key challenges including the uncertainty of customers' behavior and electricity prices in the market. To optimize their scheduling problem, riskbased programing has been advised for DRAs [11] and EVAs [12] to deal with the uncertainties.

\subsection{Literature review}

\subsubsection{Demand response aggregators}

Various DR programs have been modeled by a large number of studies. Some of the most popular DR programs, which have been implemented on the demand side, include building energy management [13], controlling cooling and heating demands [14], implementing new tariff designs [15], and designing an IncentiveBased Program (IBP) [16]. Recently, some researchers have focused on the effects of DRAs as an active player in modern power systems to apply DR programs in a lumped volume $[17,18]$. These studies have not considered the way how aggregators calculate their DR from customers. In [19], both sides of supply and demand were considered. In this paper, the interaction among the ISO, DRAs, and households was modeled based on a hierarchical market. Fang et al. [20] proposed a coupon-based DR program. This study considered the fluctuation of wind power generation and the uncertainty of real-time market prices. Wei et al. [21] proposed a two-stage bi-level framework in the presence of DR programs for the optimal scheduling of retailers by assuming the uncertainties associated with market prices and the consumption patterns of end-users. Jiang and Qian [22] used a multi-agent model to investigate the consumption behaviors of customers due to the implementation of TOU programs and level of electricity prices in the market. The risk-based scheduling was proposed in some papers including the referenced study [23] that employed the Conditional Value-at-Risk (CVaR) strategy. Scenariobased scheduling was utilized to model uncertainty in this paper. Vahid-Ghavidel et al. [24] applied IGDT to model the optimal framework for the self-scheduling of DRAs in the risk-averse environment. The uncertainty of electricity prices in the day-ahead and balancing markets was modeled by IGDT method. Moreover, the uncertainty of customer participation factors in DR programs was also simulated by IGDT strategy. Majidi et al. [25] presented a comprehensive review paper about the application of IGDT optimization method in power systems.

\subsubsection{Eclectic Vehicles Aggregator (EVA)}

In recent years, many studies in the area of EVs have focused on the scheduling problem of EVs' aggregation from different points of view including the impacts of EVs' high penetration in the market [26-28], investigation of uncertainty-based models to simulate EV owners' behavior and market prices [29,30], and risk-based approach [31,32]. Carpinelli et al. [33] proposed a multi-objective function to optimize the operation of micro grids in the presence of a large number of EVs and renewable resources. A dynamic scheduling framework was introduced [34] to find the optimal operation point of EVAs. The batteries could be charged with various electricity prices from the local distributed generators and grid. Jannati and Nazarpour [35] proposed a bi-objective function to solve the optimal scheduling problem of a parking lot equipped with solar panels. Furthermore, to respond to the environmental problems and economic issues, demand response programs were utilized. A coordinated charging framework was suggested by $\mathrm{Xu}$ and Wong [36] to decrease charging cost and power losses for various electricity loads. Alipour et al. [32] proposed the stochastic optimal scheduling of EVAs in day ahead and ancillary service markets. In this work, the uncertain nature of market prices and EVs' availability to respond to the reserve market were taken into account. Moreover, a risk-based method, Conditional Value at Risk (CVaR), was used to guarantee the upper level of the profit. Zhao et al. [37] applied the IGDT 
approach developed by Ben-Haim [38] to consider the uncertainty of market prices through the ellipsoidbound uncertainty model. However, the current study proposes a hybrid stochastic and IGDT method to consider the uncertainties. The scenario-based model is used to represent the uncertainty of the driving pattern of EV owners.

\subsection{Contribution}

One of the most important challenges to optimally schedule both DRAs and EVAs is to deal with the effective uncertainties associated with customers' behaviors and the electricity market prices. Scenariobased strategies have been widely employed in different papers to model elements of uncertain nature in the problem. The overt disadvantage of scenario-based strategies is the heavy computational burden to find a global optimal solution to the problem by increasing the number of scenario-based parameters. To the best of the authors' knowledge, no similar framework for the cooperative scheduling problem of EVAs and DRAs has been proposed in the previous literature. The main contribution of this study is to propose a risk-based framework for the cooperative scheduling problem of EVs and DR aggregators. The objective function of the problem is to maximize their profit in the day-ahead market in the uncertain decision environment. The proposed IGDT optimization approach is considered as a promising approach in not only achieving the highquality solutions but also reducing the computational burden of optimization problems. The main contributions of this work can be summarized as follows:

1. Cooperation framework of EVAs and DRAs is proposed with a risk-based strategy;

2. In the proposed framework, the uncertainty of electricity prices in the market is modeled by the IGDT method;

3. Both TOU and reward-based DR programs are applied to the associated load of three different customers who have a DR contract;

4. EVs are operated in the $\mathrm{V} 2 \mathrm{G}$ mode and TOU program is applied to EVs;

5. The opportunity and robustness functions are derived based on the market price uncertainty to provide risk-seeking and risk-averse strategies.

\subsection{Paper organization}

The rest of the paper is organized as follows. Section 2 presents the mathematical modeling of the scheduling problem of DRAs and EVAs based on IGDT method. Section 3 presents the results of an application case. Finally, the conclusion drawn from this paper is provided in Section 4.

\section{Mathematical formulation}

In this section, the mathematical formulation of the proposed framework is presented. In the first stage, the deterministic formulation of the problem is assumed without considering uncertainty of market prices. Then, the uncertain nature of the electricity prices in the day-ahead market is taken into account by the IGDT model.

\subsection{Deterministic optimal scheduling of $D R A$}

The objective of the DRA is to specify the optimal amount of DR to trade in the day-ahead market, while the DRA acts as a price taker player. First, the formulations are extracted with the assumption that the forecasted values for the electricity prices in the market are completely accurate. The profit function can be calculated through Eq. (1) and is subject to the following constraints:

$$
\text { Profit }_{0}^{D R A}=\operatorname{Max} \sum_{t=1}^{T} \tilde{\lambda}_{t}^{D A} \cdot P_{t}^{D R}-\sum_{t=1}^{T} \operatorname{Cost}_{t}^{D R},
$$

S.t.:

$$
\begin{aligned}
& P_{t}^{D R}=\sum_{c \in N_{c}} P_{t, c}^{r w}+P_{t, c}^{T O U}, \quad \forall t \\
& P_{t, c}^{r w}=\sum_{s=1}^{N_{s}} P F_{t} \cdot \bar{P}_{t, c}^{r w, s} \cdot u_{t, c}^{r w, s}, \quad \forall t, \forall c, \forall s \\
& R_{t, c}^{r w}=\sum_{s=1}^{N_{s}} R_{t, c}^{r w, s}, \quad \forall t, \forall c, \forall s, \\
& \bar{R}_{t, c}^{r w, s-1} \cdot u_{t, c}^{r w, s} \leq R_{t, c}^{r w, s} \leq \bar{R}_{t, c}^{r w, s} \cdot u_{t, c}^{r w, s}, \\
& \sum_{s=1}^{N_{s}} u_{t, c}^{r w, s} \leq 1, \quad \forall t, \forall c, \forall s, \\
& \operatorname{Cost}_{t}^{D R}=\sum_{c=1}^{N_{c}} \sum_{s=1}^{N_{s}} R_{t, c}^{r w, s} \cdot P F_{t, c} \cdot P_{t, c}^{r w, s}, \\
& P_{t, c}^{T O U}= \\
& P_{\min } \leq P_{t}^{D R} \leq P_{\max }, \\
& -\operatorname{Load}_{t, c}^{0} \cdot \sum_{h=1}^{T}\left(e_{c}(t, h) \cdot \frac{\left(\lambda_{T O U}(t, h)-\lambda_{f i x}(t, h)\right)}{\lambda_{f i x}(t, h)}\right.
\end{aligned}
$$

Eq. (1) presents the deterministic model of the DRA's profit maximization problem, which is equal to the revenue coming from trading DR minus the imposed cost due to the implementation of the rewardbased DR program. Terms $\tilde{\lambda}_{t}^{D A}$ and $P_{t}^{D R}$ depict the forecasted day-ahead price and the volume of $D R$, respectively. The total DR power, $P_{t}^{D R}$, is derived 


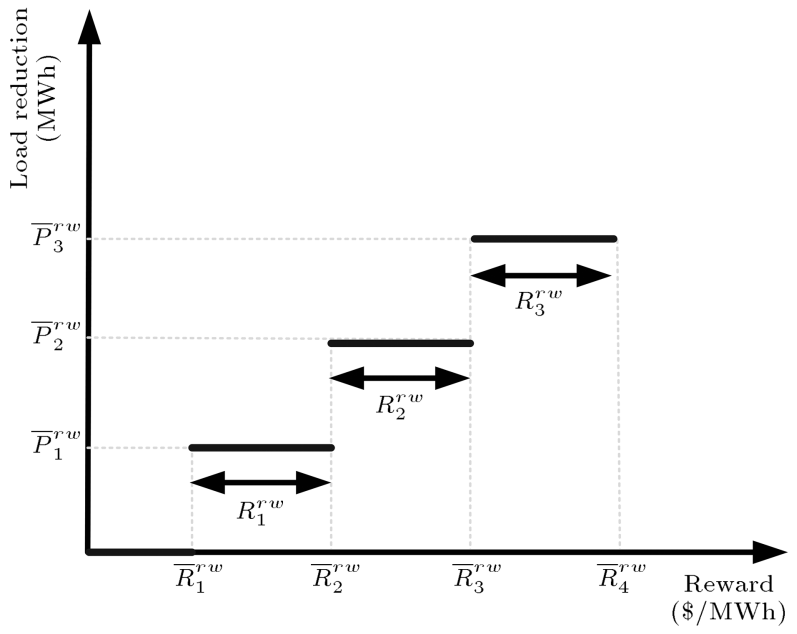

Figure 1. Reward-based DR curve.

from the application of the reward-based and TOU programs, as indicated in Eq. (2). The reward-based DR power $P_{t, c}^{r w}$ of each customer $c$ at hour $t$ is calculated by Eq. (3) at the power level of each step $s$, where $P_{t, c}^{r w}$ and $\bar{P}_{t, c}^{r w, s}$ refer to the total load reduction and the steps of reduced load through enforcing the rewardbased DR program for consumer $c$ at hour $t$. Term $P F_{t}$ is the participation factor of consumers, which is considered constant in this study. Furthermore, the binary variables $\alpha_{t, c}^{r w, s}$ determine that DRA can only choose one level of the offered load reduction $s$, as presented in Eq. (6). Eq. (4) explains the summation of reward for each customer at hour $t, R_{t, c}^{r w}$, and Eq. (5) limits the amount of reward for each step between the amount of the reward for the current step, $\bar{R}_{t, c}^{r w, s}$, and that for pervious step, $\bar{R}_{t, c}^{r w, s-1}$. In Figure 1 , the amount of load reduction, $\bar{P}_{t, c}^{r w, s}$, and the related reward, $\bar{R}_{t, c}^{r w, s}$, are portrayed by the vertical and horizontal axes of the figure. Finally, the cost of reward-based DR program, Cost $_{t}^{D R}$, at hour $t$ can be calculated due to Eq. (7).

The acquired power by applying TOU program can be calculated by Eq. (8). Unlike the rewardbased program, the TOU program does not impose any cost on the DRA and just suggests distinct tariffs instead of incentives paid to consumers. According to Eq. (4), consumers receive different price tariffs related to the consumption time like off-peak and peak tariffs. In Eq. (8), $\lambda_{f i x}(h, t)$ depicts the initial price associated with consumer $c$ at interval $h$ while $\lambda_{\text {TOU }}(t, h)$ represents the offered TOU price. Term $e_{c}(t, h)$ shows the price elasticity of consumer $c$ at hour $t$ compared to period $h$.

\subsubsection{Deterministic optimal scheduling of EVA}

As presented in Eq. (10), the objective function of the EVA is to maximize its profit in the day-ahead market. In deterministic scheduling, only one driving pattern is considered so that the daily consumption energy is equal to the expected value. According to Eq. (10), it is assumed that EVA can perform EVs in the Vehicle to Grid (V2G) state, i.e., it can not only buy power from the market to charge EVs' batteries, but also sell the saved power in the batteries due to the EV owners' driving patterns.

$$
\begin{aligned}
\operatorname{Profit}_{E V A}^{0}= & \operatorname{Max} \sum_{t=1}^{T} \sum_{v=1}^{N_{v}} N_{E V} \cdot \tilde{\lambda}_{t}^{D A} \cdot\left(P_{v, t}^{\text {disch }}-P_{v, t}^{c h}\right) \\
& +\sum_{t=1}^{T} \sum_{v=1}^{N_{v}} N_{E V}, P_{v, t}^{0} \cdot \lambda_{\text {fixed }}^{E V},
\end{aligned}
$$

s.t.:

$$
\begin{aligned}
& S O C_{v, t}=S O C_{v, t-1}+\eta_{c h}\left(P_{v, t}^{c h} / \overline{P_{v}^{c h}}\right)-1 / \eta_{\text {disch }} \\
& \cdot\left(P_{v, t}^{d i s c h} / \overline{P_{v}^{d i s c h}}\right)-P_{v, t}^{0} \\
& \underline{S O C_{v}} \leq S O C_{v, t} \leq \overline{S O C_{v}} \\
& \underline{P_{v}^{c h}} \cdot u_{v, t}^{c h} \leq P_{v, t}^{c} \leq \overline{P_{v}^{c h} \cdot} u_{v, t}^{c h}, \\
& \underline{P_{v}^{d i s c h}} \cdot u_{v, t}^{\text {disch }} \leq P_{v, t}^{\text {disch }} \leq \overline{P_{v}^{\text {disch }}} \cdot u_{v, t}^{\text {disch }}, \\
& u_{v, t}^{c h}+u_{v, t}^{\text {disch }} \leq 1 \text {. }
\end{aligned}
$$

In Eq. (10), the terms $P_{v, t}^{d i s c h}$ and $P_{v, t}^{c h}$ stand for discharging and charging power rates of a vehicle with driving pattern $v$ at time $t$, respectively. The amount of power consumed by each EV at time is depicted by $P_{v, t}^{0}$. EVAs attempt to provide power with economical prices to encourage EV owners to sign a contract with them. In this regard, the value of $\lambda_{\text {fixed }}^{E V}$ is considered lower than retailer price of power sale to customers. Eq. (11) is used to calculate $S O C_{v, t}$, showing the state of charge vehicle in an hourly format. In order to force the minimum and maximum levels of $S O C_{v, t}$, Relation (12) must be fulfilled. In Relations (13) and (14), the binary variables $u_{v, t}^{c h}$ and $u_{v, t}^{\text {disch }}$ denote the states of charge and discharge, respectively. Constraint (15) enforces a restriction so that each EV can only be charged or discharged. It is worth mentioning that the presented equations are formulated for the stochastic scheduling. To avoid rewriting the equations, they can also be used for the deterministic scheduling, while the number of driving pattern $V$ is equal to 1 .

\subsubsection{Cooperation of DRA and EVA}

In this section, the proposed framework for DR trading is presented in Figure 2. The case study is a load with different electricity consumers including residential, commercial, and industrial sectors, as well as the demand of EVs provided by an EVA. For each sector, the amount of TOU tariff and reward-based DR is 


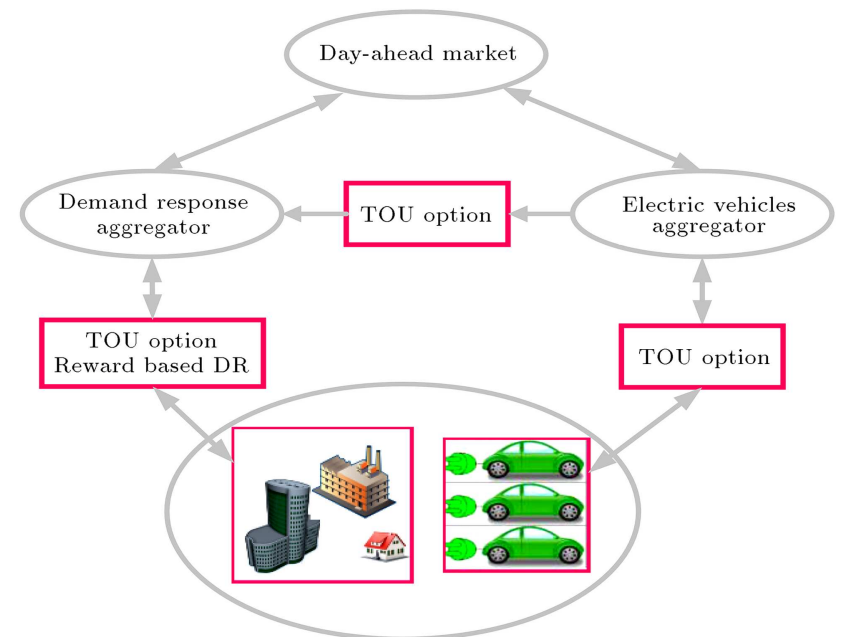

Figure 2. Cooperative scheduling framework between DRA and EVA.

offered individually. As shown in Figure 2, through the double-sided arrows, the energy can flow from consumers to DR purchasers, and vice versa.

It is implied that while the DRA obtains DR to sell to buyers at peak times, customers are encouraged to consume more electricity energy during offpeak times. Furthermore, the DRA and EVA have a cooperation contract for TOU program. In other words, EVA can receive a portion of the profit that DRA earns by trading the TOU load. As a result, in this case, the EV time of use load and total DR power can be found by Eqs. (16) and (17), respectively.

$$
\begin{aligned}
& P_{v, t}^{T O U}= \\
& -P_{v, t}^{0} \cdot \sum_{h=1}^{T}\left(e_{E V}(t, h) \cdot \frac{\left(\lambda_{E V}^{T O U}(t, h)-\lambda_{f i x}^{E V}(t, h)\right)}{\lambda_{f i x}^{E V}(t, h)}\right), \\
& P_{t}^{D R}=\sum_{c \in N_{c}} P_{t, c}^{r w}+P_{t, c}^{T O U}+\sum_{v=1}^{N_{v}} N_{E V} \cdot P_{v, t}^{T O U}, \quad \forall t
\end{aligned}
$$

\section{Information-Gap Decision Theory (IGDT)}

In this study, IGDT scheduling framework is proposed and formulated to model the error between the real and forecasted values of the uncertain parameters, while the objective function is to maximize the expected profit. Second, by applying the proposed framework, aggregators can face price oscillation with two different approaches: risk-seeking and risk-averse approaches.

The parameters modeled by the IGDT method are the day-ahead prices, which are of uncertain nature. In the current work, the DRA takes part in the day-ahead market, and forecasted electricity prices, stranded by $\tilde{\lambda}=\left[\tilde{\lambda}_{1}, \tilde{\lambda}_{2}, \ldots, \tilde{\lambda}_{T}\right]$, are assumed to be accessible.
Envelope bound of the IGDT model will be used to simulate the uncertainty as follows:

$$
U\left(\tilde{\lambda}_{t}, \alpha\right)=\left\{\lambda: \frac{\left|\lambda_{t}-\tilde{\lambda}_{t}\right|}{\tilde{\lambda}_{t}} \leq \alpha\right\}, \quad \alpha \geq 0
$$

where $\alpha$ denotes the horizon of the uncertain parameters. Moreover, $\lambda_{t}$ and $\tilde{\lambda}$ depict the real and forecasted electricity prices at hour $t$, respectively. In this model, the forecasted day-head prices are input, while the horizon of the uncertain parameters is computed through the decision-making procedure. Note that the lower and upper limits of variable $\alpha$ will be determined in the solution process.

In the proposed model, the DRAs gain power from the DR customers to do their commitment in the dayahead market. The aggregator encourages customers to sell power during pick hours and shift their energy consumption to off-pick hours by offering TOU tariffs and appropriate incentives. As a result, the expected profit of DRA depends on electricity prices in the market.

\subsection{Robust scheduling}

The risk-averse approach aims to schedule the operation of DRA so that the profit is guaranteed to be higher than or equal to a critical profit due to the undesirable fluctuation of price from the forecasted value. It is then formulated as follows:

$$
\widehat{\alpha}\left(P, B_{r}\right)=\operatorname{Max}_{P}\left\{\alpha_{r}: \operatorname{Min}_{\lambda_{t}} B\left(P, \lambda_{t}\right) \geq B_{r}\right\},
$$

where $P$ and $B_{r}$ stand for decision variables vector and the target profit, respectively. In the current work, the amount of trading DR at each hour is the decision variable. The intention of the robustness function, $\widehat{\alpha}\left(P, B_{r}\right)$, is to find the maximum uncertainty horizon such that the critical profit is obtained in the worst case and all constraints are fulfilled. This means that the IGDT model aims to maximize the profit in the region of the uncertainty while the expected total profit of DRA is achieved. Note that the critical profit is a proportion of the risk-neutral profit. Due to Eq. (19) and presented statement, a greater amount of $\alpha_{r}$ is more desirable. Hence, the robust scheduling formulation for the cooperative scheduling problem of DRA and EVA can be formulated as follows:

$$
\operatorname{Max}_{P} \alpha_{r}
$$

s.t.:

$$
\text { Profit }_{\text {total }}^{*} \geq B_{r}=\text { Profit }_{\text {total }}^{0}(1-d),
$$




$$
\begin{aligned}
\operatorname{Profit}_{\text {total }}^{*}= & \operatorname{Min}_{\Delta \lambda_{t}}\left(\sum_{t=1}^{T}\left(\tilde{\lambda}_{t}+\Delta \lambda_{t}\right) \cdot P_{t}^{D R}\right. \\
& -\sum_{t=1}^{T} \operatorname{Cost}_{t}^{D R}+\sum_{t=1}^{T} \sum_{v=1}^{N_{v}}\left(\tilde{\lambda}_{t}^{D A}+\Delta \lambda_{t}\right) \\
& .\left(P_{v, t}^{d i s c h}-P_{v, t}^{c h}\right)+\sum_{t=1}^{T} \sum_{v=1}^{N_{v}} P_{v, t}^{T O U} \\
& \left.. \lambda_{T O U}^{E V}\right),
\end{aligned}
$$

s.t.:

$$
-\alpha \tilde{\lambda}_{t} \leq \Delta \lambda_{t} \leq \alpha \tilde{\lambda}_{t}
$$

Note that the proposed IGDT method is generally known as bi-level, which can be solved through the common bi-level models as explained [39]. In addition, in certain circumstances, the IGDT-based models can be divided into two single level problems [27]. In the proposed scheduling problem, decreasing electricity price has a negative impact on the scheduling profit. In other words, if the market price drops, then the scheduling profit will increase as well, or vice versa; if the electricity price decreases, the profit will certainly decrease. Thus, the proposed IGDT model can be converted into the single-level problem. Since the sale of energy is higher than the purchased energy in this problem, increasing market price has a positive effect on the scheduling profit. Therefore, the proposed bilevel scheduling problem can be converted to a singlelevel problem.

\subsection{Opportunistic scheduling}

When the presented problem follows a risk-seeking approach, the variable decisions are specified with an optimistic attitude in order to have a positive effect on the profit. Due to the opportunity function, the lower favorable price deviation is found desirable, whereas the earned profit is higher than the risk-neutral state. This can be represented as follows:

$$
\widehat{\beta}\left(P, B_{o p}\right)=\operatorname{Min}_{P}\left\{\alpha_{o p}: \operatorname{Max}_{\lambda_{t}} B\left(P, \lambda_{t}\right) \geq B_{o p}\right\} .
$$

Term $B_{o p}$ shows the pre-determined profit for the opportunity approach. The objective function of the opportunity strategy, $\widehat{\beta}\left(P, B_{o p}\right)$, attempts to calculate the minimum price deviation to obtain higher profit than $B_{o p}$ and fulfills the constraints, too. As a result, the opportunity function for cooperation of DRA and EVA can be follows:

$$
\operatorname{Min}_{P} \alpha_{o p}
$$

s.t.:

$$
\text { Profit }_{\text {total }}^{*} \geq B_{o p}=\text { Profit }_{\text {total }}^{0}(1+d),
$$

$$
\begin{aligned}
\operatorname{Profit}_{\text {total }}^{*}= & \operatorname{Max}_{\Delta \lambda_{t}}\left(\sum_{t=1}^{T}\left(\tilde{\lambda}_{t}+\Delta \lambda_{t}\right) \cdot P_{t}^{D R}\right. \\
& -\sum_{t=1}^{T} \operatorname{Cost}_{t}^{D R}+\sum_{t=1}^{T} \sum_{v=1}^{N_{v}}\left(\tilde{\lambda}_{t}^{D A}+\Delta \lambda_{t}\right) \\
& \left(P_{v, t}^{\text {disch }}-P_{v, t}^{c h}\right)+\sum_{t=1}^{T} \sum_{v=1}^{N_{v}} P_{v, t}^{T O U} \\
& \left.. \lambda_{\text {fixed }}^{\text {EV }}\right)
\end{aligned}
$$

s.t.:

$-\alpha \tilde{\lambda}_{t} \leq \Delta \lambda_{t} \leq \alpha \tilde{\lambda}_{t}$.

As explained similarly for the robustness function, the opportunity optimization function is also solved as a bi-level problem. Therefore, the value of the uncertainty horizon variable, $\alpha_{o p}$, is minimized at the upper level, while the amount of decision variables is determined at the lower level in order to guarantee the expected profit higher than pre-determined profit $B_{o p}$. In this strategy, the decision-maker acts riskily. As mentioned in Subsection 3.1, the amount of the purchased power is lower than that of sale power, i.e., the maximum profit in Eq. (27) is readily seen to occur at the highest price.

\section{Results and discussions}

\subsection{Data}

The proposed programing model is coded in Mixed Integer Non-Linear Programming (MINLP) under the General Algebraic Modeling System (GAMS). It is worth mentioning that the proposed bi-level optimization is converted to a single-level problem, as mentioned in Section 3. The adopted load information of DRA's customers includes residential, commercial, and industrial sectors, and forecasted day-ahead prices are depicted in Figure 3. The elasticity of the different lodes and retailer TOU prices are reported in Tables 1 and 2 , respectively. To model the uncertainty of the driving pattern, 1000 scenarios are generated via Mont Carlo simulation. Then, SCENRED tool under GAMS environment is employed to decrease the number of the scenarios with respect to their probability into 10 driving patterns [40].

According to Figure 1, only 5 steps for the rewardbased DR program are considered. Since EVA can perform EVs in the $\mathrm{V} 2 \mathrm{G}$ state, there is no economic 


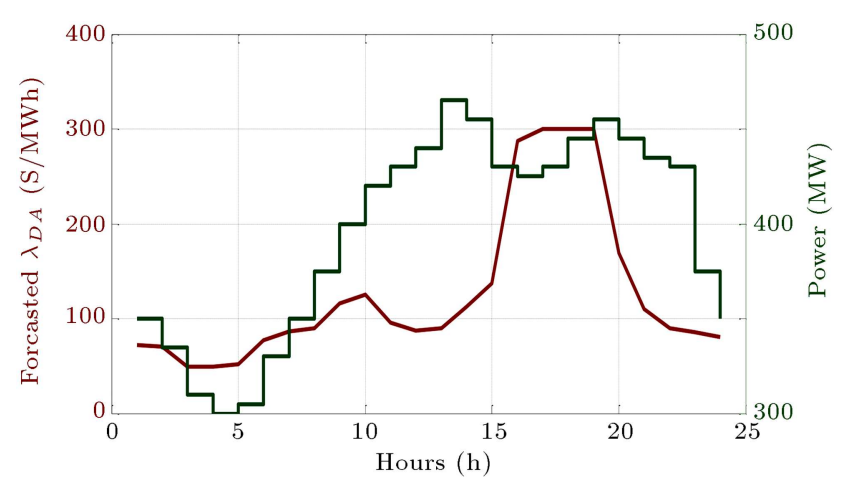

Figure 3. Adapted forecast day-ahead price and load curve.

Table 1. The retailer $T O U$ prices.

\begin{tabular}{|c|c|c|c|}
\hline & & $\lambda_{f i x}(t, h)$ & $\lambda_{T O U}(t, h)$ \\
\hline \multirow[t]{2}{*}{ Residential } & Peak & 294 & 346 \\
\hline & Off-peak & 294 & 213 \\
\hline \multirow[t]{2}{*}{ Commercial } & Peak & 255 & 281 \\
\hline & Off-peak & 255 & 205 \\
\hline \multirow[t]{2}{*}{ Industrial } & Peak & 331 & 424 \\
\hline & Off-peak & 331 & 135 \\
\hline
\end{tabular}

Table 2. Elasticity matrices of different customers [24,40].

\begin{tabular}{lccc}
\hline \multirow{2}{*}{ Residential } & & Peak & Off-peak \\
\hline \multirow{2}{*}{ Commercial } & Peak & -0.15 & -0.05 \\
\cline { 2 - 4 } & Off-peak & 0.02 & -0.03 \\
\cline { 2 - 4 } & Peak & -0.16 & 0.06 \\
\hline \multirow{2}{*}{ Ondustrial } & Peak & -0.2 & 0.1 \\
\cline { 2 - 4 } & Off-peak & 0.07 & -0.08 \\
\hline Electric vehicle & Peak & -0.15 & 0.05 \\
\cline { 2 - 4 } & Off-peak & 0.02 & -0.03 \\
\hline
\end{tabular}

incentive for it to have a contract with DRA to participate in the reward-based DR program. Therefore, EVA participates only in the TOU program.

\subsection{Case study}

\subsubsection{Deterministic scheduling}

In this subsection, the numerical results of the scheduling problem are provided without considering the uncertainty of the day-ahead prices for different cases including 1) individual DRA and EVA scheduling, and 2 ) cooperative scheduling of DRA and EVA. Note that if the EVA cooperates with DRA, it can receive a fraction of the profit that the DRA gains due to the application of the TOU-based DR program to EVs. In this study, it is assumed that this profit is equally divided between the two aggregators. The profit of each case is reported in Table 3 . In this case, only one driving pattern is considered.

According to Table 3, when EVA and DRA have a cooperative contract about TOU load of EVs, they can increase their profit without imposing any additional cost. The profit of EVA can increase by about $13 \%$ compared with individually scheduling state. Additionally, DRA's profit has risen by $\$ 388.31$ through the given period, which is 24 hours, and this rise in the profit can be a considerable amount of money during a year. Of note, for solving the scheduling problem through the IGDT method, the cooperative scheduling of DRA and EVA is considered.

\subsubsection{IGDT-based scheduling}

In this case, the IGDT-based model is used to show the effects of market price uncertainty on the profit. According to the IGDT model, there are two different attitudes: risk-averse and risk-seeking attitudes. First, the scheduling problem is solved for the risk-averse attitude with different profit deviation factors that increased from $d=0.05$ to $d=0.3$. The robustness function values are depicted for both DRA and EVA in Figures 4 and 5, respectively. Note that, in this case, different driving pattern scenarios are produced.

As expected, the value of the robustness function for both aggregators raises by increasing the deviation factor while the profit decreases. In other words, if the aggregators would like to make a more riskaverse decision, less profit can be guaranteed. For example, when the deviation factor is 0.3 (the worst

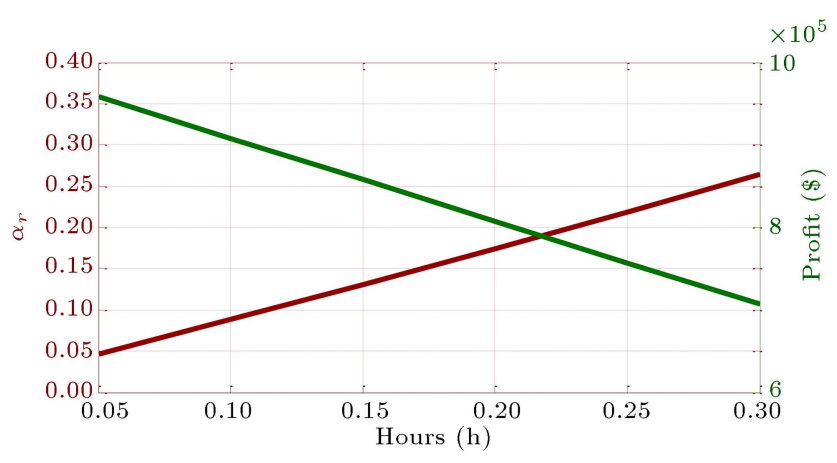

Figure 4. Robustness function of $\alpha$ versus $d$ for DRA.

Table 3. Profit for individual and cooperative scheduling.

\begin{tabular}{ccccc}
\hline & \multicolumn{2}{c}{ Individually } & scheduling & \multicolumn{2}{c}{ Cooperative scheduling } \\
\cline { 2 - 5 } & DRA & EVA & DRA & EVA \\
\hline Profit $(\$)$ & 1012307.313 & 3596.000 & 1012695.622 & 4081.386 \\
\hline
\end{tabular}


Table 4. Optimal opportunity function for DRAs and EVAs.

\begin{tabular}{ccccc}
\hline Deviation & Profit $_{\text {total }}^{*}(\$)$ & Profit $_{\boldsymbol{D} \boldsymbol{A} \boldsymbol{A}}(\$)$ & Profit $_{E V A}(\$)$ & $\boldsymbol{\alpha}_{\boldsymbol{O} \boldsymbol{p}}$ \\
\hline 0.0 & 1015949.984 & 1011821.869 & 5110.291 & 0.0 \\
0.05 & 1063942.110 & 1058596.069 & 5346.041 & 0.038 \\
0.1 & 1114606.020 & 1109157.571 & 5448.449 & 0.079 \\
0.15 & 1165269.930 & 1159722.839 & 5547.091 & 0.118 \\
0.2 & 1215933.840 & 1210289.262 & 5644.578 & 0.156 \\
0.25 & 1266597.750 & 1260856.566 & 5741.184 & 0.195 \\
0.3 & 1317261.660 & 1311424.372 & 5837.288 & 0.233 \\
\hline
\end{tabular}

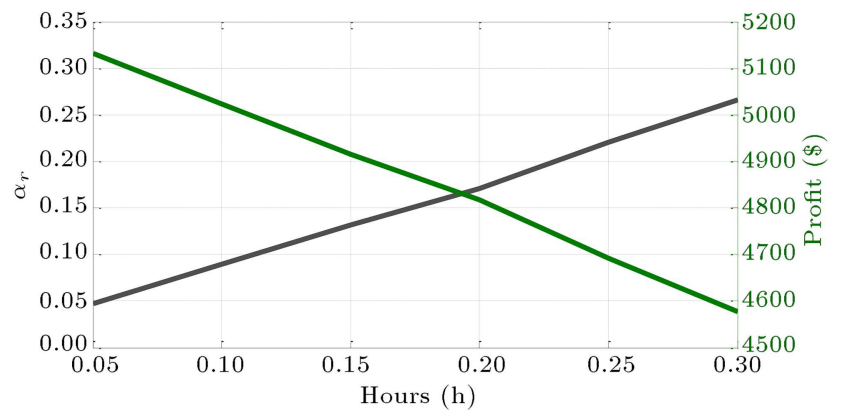

Figure 5. Robustness function of $\alpha$ versus $d$ for EVA.

case), the critical total profit is equal to $\$ 709294.740$. The profits of DR and EV aggregators are equal to $\$ 704716.762$ and $\$ 4577.978$, respectively. Based on the IGDT scheduling, this amount of the profits is achieved only when the error of electricity price in the market is no more than $\alpha_{r}=0.266$ or $26.6 \%$.

The optimum opportunity function values are optimized for pursuing risk-seeking attitudes by DRAs and EVAs. The results illustrated in Table 4 indicate that higher desired market price errors from the forecast values are essential to achieving a higher target profit. For instance, to reach a total profit $25 \%$ higher than Profit total, there must be at least $14.8 \%$ error between the forecasted prices and observed values. For example, for $d=0.3$, to achieve a total profit higher than Profit ${ }_{\text {total }}^{*}=\$ 1317261.660$, hourly errors must be higher than $23.3 \%$.

\section{Conclusion}

This study evaluated the cooperation of DRA and EVA with respect to the uncertainty of electricity price and EV owners' driving behavior in the day ahead-market. To simulate the uncertainties, IGDT, risk-based approach, and scenario-based method were applied to the proposed cooperative framework. The DRA trades DR, which is supplied through the implementation of two different DR programs on its customers including reward-based DR and TOU programs. On the other hand, EVA could increase its profit by trading V2G and setting a TOU contract with DRA. To evaluate the proposed method, a case study was analyzed. The scheduling problem was solved for both of the controversial strategies called risk-averse strategy and risk-seeking strategy. The profit of both aggregators increased while in cooperation. Generally, the results showed the effectiveness of the proposed framework.

\section{Nomenclature}

\section{Sets and indices}

$t \quad$ Index of time, $t=\{1,2,3, \ldots, T\}$

$D R \quad$ Index of DR program including $D R_{t}=\left\{r w_{t}, T O U_{t}\right\}$ at hour $t$

c Index of consumer including

$c=\{$ Res, Com, Ind $\}$

Res Residential consumer

Com Commercial consumer

Ind Industrial consumer

Parameters and constants

$\overline{P_{v}^{d}} / P_{v}^{d} \quad$ Maximum $/$ minimum discharge rate of $\mathrm{EV} v$

$\tilde{\lambda}_{t}^{D} \quad$ Forecasted electricity price in day-

ahead market at hour $t$

$\bar{P}_{t, c}^{r w, s} \quad$ Reduced load steps in the reward-based DR program at hour $t$

$P_{\min } / P_{\max } \quad$ Maximum $/$ minimum capacities of DRA

$\operatorname{Load}_{t, c}^{0} \quad$ Initial demand of consumer $c$ at hour $t$

$e_{c}(h, t) \quad$ Elasticity of consumer $c$ at hour $t$ related to price at hour $h$

$\lambda_{f i x}(h, t) \quad$ Fixed rate price

$\lambda_{\text {TOU }}(h, t) \quad$ TOU price

$P F_{t} \quad$ Participation factors of consumers in the reward-based DR program at hour $t$ 
$\bar{R}_{t, c}^{r w, s}$

Determined reward for consumer $c$ at hour $t$ in the reward-based DR program

$\overline{P_{v}^{c h}} / \underline{P_{c}^{c h}} \quad$ Maximum $/$ minimum charge rate of PEV $v$

$\overline{S O C_{v}} / \underline{S O C_{v}}$ Maximum/minimum level of SOC of $\mathrm{EV} v$

$\eta_{c} / \eta_{\text {dis }}$ Charging/discharging rate of $\mathrm{PEV}$

\section{Function}

$U\left(\tilde{\lambda}_{t}, \alpha\right)$

Uncertainty model in the IGDT approach

$\hat{\alpha}\left(P, B_{r}\right)$

$\hat{\beta}\left(P, B_{o p}\right)$

\section{Variables}

$\alpha$

$\lambda_{t}^{D}$

$P_{t}^{D}$

$P_{t}^{D R}$

$P_{t, c}^{r w}$

$P_{t, c}^{T O U}$

$\operatorname{Cost}_{t}^{D R}$

Profit $_{0}^{D R A}$

Profit $_{E V A}^{0}$

$R_{t, c}^{r w, s}$

$\alpha_{r}$

$\alpha_{o p}$

$P$

$S O C_{v, t}$

$P_{v, t}^{c h}$

Horizon of the uncertain parameter

Electricity price in day- ahead market at hour $t$

Offered power in the day-ahead market at hour $t$

Total power reduction in the result of the DR programs at hour $t$

Power reduction of consumer $c$ in the result of the reward-based DR program at hour $t$

Power reduction of consumer $c$ in the result of the TOU program at hour $t$

Cost of the aggregator due to the DR programs at hour $t$

Profit of the DRA in deterministic scheduling

Profit of the EVA in deterministic scheduling

The reward paid to consumer $c$ in the reward-based DR program

Optimal robustness function value

Optimal opportunity function value

Decision variables of the IGDT model

SOC of EV $v$ at time $t$

Charge power of EV $v$ at time $t$

\section{Binary variables}

$u_{t, c}^{r w, s}$

Binary variable of the reduced load for step $s$ in the reward-based DR at hour $t$

$u_{v, t}^{c h} / u_{v, t}^{\text {disch }}$
Binary variable of charge/discharge state

\section{References}

1. Gadham, K.R. and Ghose, T. "Importance of social welfare point for the analysis of demand response", in IEEE First International Conference on Control, Measurement and Instrumentation (CMI), pp. 182-185 (2016).

2. Aalami, H.A. and Khatibzadeh, A. "Regulation of market clearing price based on nonlinear models of demand bidding and emergency demand response programs", International Transactions on Electrical Energy Systems, 26(11), pp. 2463-2478 (2016).

3. Huang, S., Wu, L., Infield, D., and Zhang, T. "Using electric vehicle fleet as responsive demand for power system frequency support", In 2013 IEEE Vehicle Power and Propulsion Conference (VPPC), pp. 1-5 (2013).

4. Zhao, F., Liu, F., Liu, Z., and Hao, H. "The correlated impacts of fuel consumption improvements and vehicle electrification on vehicle greenhouse gas emissions in China", Journal of Cleaner Production, 207, pp. 702716 (2019).

5. Shamshirband, M., Salehi, J., and Gazijahani, F.S. "Decentralized trading of plug-in electric vehicle aggregation agents for optimal energy management of smart renewable penetrated microgrids with the aim of $\mathrm{CO}_{2}$ emission reduction", Journal of Cleaner Production, 200, pp. 622-640 (2018).

6. Schneider, K., Gerkensmeyer, C., Kintner-Meyer, M., and Fletcher, R. "Impact assessment of plug-in hybrid vehicles on pacific northwest distribution systems", in Power and Energy Society General MeetingConversion and Delivery of Electrical Energy in the 21st Century, pp. 1-6 (2008).

7. Kintner-Meyer, M., Schneider, K., and Pratt, R., Impacts Assessment of Plug-in Hybrid Vehicles on Electric Utilities and Regional US Power Grids, Part 1: Technical Analysis, Pacific Northwest National Laboratory, 1 (2007).

8. Zhili, D., Boqiang, L., and Chunxu, G. "Development path of electric vehicles in China under environmental and energy security constraints", Resources, Conservation and Recycling, 143, pp. 17-26 (2019).

9. Kempton, W. and Dhanju, A. "Electric vehicles with V2G", Windtech International, 2(2), p. 18 (2006).

10. Bessa, R.J. and Matos, M. "Global against divided optimization for the participation of an EV aggregator in the day-ahead electricity market. Part I: Theory", Electric Power Systems Research, 95, pp. 309-318 (2013).

11. Mahmoudi, N., Saha, T.K., and Eghbal, M. "A new trading framework for demand response aggregators", In 2014 IEEE PES General Meeting-Conference \& Exposition, pp. 1-5 (2014).

12. Zhao, J., Wan, C., Xu, Z., and Wang, J. "Risk-based day-ahead scheduling of electric vehicle aggregator using information gap decision theory", IEEE Transactions on Smart Grid, 8(4), pp. 1609-1618 (2015). 
13. González, R.M., et al. "Optimizing electricity consumption of buildings in a microgrid through demand response", In 2017 IEEE Manchester PowerTech, pp. 1-6 (2017). DOI:10.1109/PTC.2017.7980983

14. Wai, C.H., Beaudin, M., Zareipour, H., Schellenberg, A., and Lu, N. "Cooling devices in demand response: A comparison of control methods", IEEE Transactions on Smart Grid, 6(1), pp. 249-260 (2014).

15. Nguyen, D.T., Nguyen, H.T., and Le, L.B. "Dynamic pricing design for demand response integration in power distribution networks", IEEE Transactions on Power Systems, 31(5), pp. 3457-3472 (2016).

16. Kim, D.-M. and Kim, J.-O. "Design of emergency demand response program using analytic hierarchy process", IEEE Transactions on Smart Grid, 3(2), pp. 635-644 (2012).

17. Liu, G. and Tomsovic, K. "A full demand response model in co-optimized energy and reserve market", Electric Power Systems Research, 111, pp. 62-70 (2014).

18. Parvania, M. and Fotuhi-Firuzabad, M. "Demand response scheduling by stochastic SCUC", IEEE Transactions on Smart Grid, 1(1), pp. 89-98 (2010).

19. Wei, M. "A top-down market model for demand response procurement via aggregators", In 2015 IEEE Eindhoven PowerTech, pp. 1-5 (2015). DOI:10.1109/PTC.2015.7232657

20. Fang, X., Hu, Q., Li, F., Wang, B., and Li, Y. "Coupon-based demand response considering wind power uncertainty: A strategic bidding model for load serving entities", IEEE Transactions on Power Systems, 31(2), pp. 1025-1037 (2015).

21. Wei, W., Liu, F., and Mei, S. "Energy pricing and dispatch for smart grid retailers under demand response and market price uncertainty", IEEE Transactions on Smart Grid, 6(3), pp. 1364-1374 (2014).

22. Jiang, Z. and Qian, A. "Agent-based simulation for symmetric electricity market considering price-based demand response", Journal of Modern Power Systems and Clean Energy, 5(5), pp. 810-819 (2017).

23. Mahmoudi, N., Heydarian-Forushani, E., Shafie-khah, M., Saha, T.K., Golshan, M., and Siano, P. "A bottom-up approach for demand response aggregators' participation in electricity markets", Electric Power Systems Research, 143, pp. 121-129 (2017).

24. Vahid-Ghavidel, M., Mahmoudi, N., and MohammadiIvatloo, B. "Self-scheduling of demand response aggregators in short-term markets based on information gap decision theory", IEEE Transactions on Smart Grid, 10(2), pp. 2115-2126 (2019).

25. Majidi, M., Mohammadi-Ivatloo, B., and Soroudi, A. "Application of information gap decision theory in practical energy problems: A comprehensive review", Applied Energy, 249, pp. 157-165 (2019).
26. Ahmadian, A., Sedghi, M., Mohammadi-ivatloo, B., Elkamel, A., Golkar, M.A., and Fowler, M. "Costbenefit analysis of $\mathrm{V} 2 \mathrm{G}$ implementation in distribution networks considering PEVs battery degradation", IEEE Transactions on Sustainable Energy, 9(2), pp. 961-970 (2017).

27. Lin, H., Liu, Y., Sun, Q., Xiong, R., Li, H., and Wennersten, R. "The impact of electric vehicle penetration and charging patterns on the management of energy hub-A multi-agent system simulation", Applied Energy, 230, pp. 189-206 (2018).

28. Ghahramani, M., Nojavan, S., Zare, K., and Mohammadi-ivatloo, B. "Short-term scheduling of future distribution network in high penetration of electric vehicles in deregulated energy market", Operation of Distributed Energy Resources in Smart Distribution Networks, Elsevier, pp. 139-159 (2018).

29. Kim, Y., Kong, S., and Joo, S.-K. "Stochastic charging coordination method for electric vehicle (EV) aggregator considering uncertainty in EV departures" Journal of Electrical Engineering and Technology, 11(5), pp. 1049-1056 (2016).

30. Karfopoulos, E., Marmaras, C.E., and Hatziargyriou, N. "Charging control model for electric vehicle supplier aggregator", in Innovative Smart Grid Technologies (ISGT Europe) International Conference and Exhibition, pp. 1-7 (2012).

31. Momber, I., Siddiqui, A., San Román, T.G., and Söder, L. "Risk averse scheduling by a PEV aggregator under uncertainty", IEEE Transactions on Power Systems, 30(2), pp. 882-891 (2015).

32. Alipour, M., Mohammadi-Ivatloo, B., MoradiDalvand, M., and Zare, K. "Stochastic scheduling of aggregators of plug-in electric vehicles for participation in energy and ancillary service markets", Energy, 118, pp. 1168-1179 (2017).

33. Carpinelli, G., Caramia, P., Mottola, F., and Proto, D. "Exponential weighted method and a compromise programming method for multi-objective operation of plug-in vehicle aggregators in microgrids", International Journal of Electrical Power \& Energy Systems, 56, pp. 374-384 (2014).

34. Škugor, B. and Deur, J. "Dynamic programming-based optimisation of charging an electric vehicle fleet system represented by an aggregate battery model", Energy, 92, pp. 456-465 (2015).

35. Jannati, J. and Nazarpour, D. "Optimal performance of electric vehicles parking lot considering environmental issue", Journal of Cleaner Production, 206, pp. 1073-1088 (2019).

36. Xu, J. and Wong, V.W. "An approximate dynamic programming approach for coordinated charging control at vehicle-to-grid aggregator", In Smart Grid Communications (SmartGridComm) International Conference, pp. 279-284 (2011).

37. Zhao, J., Wan, C., Xu, Z., and Wang, J. "Risk-based day-ahead scheduling of electric vehicle aggregator 
using information gap decision theory", IEEE Trans. Smart Grid, 99, pp. 1-10 (2015).

38. Ben-Haim, Y., Information-Gap Decision Theory: Decisions Under Severe Uncertainty, Academic Press London (2001).

39. Shafiee, S., Zareipour, H., Knight, A.M., Amjady, N., and Mohammadi-Ivatloo, B. "Risk-constrained bidding and offering strategy for a merchant compressed air energy storage plant", IEEE Transactions on Power Systems, 32(2), pp. 946-957 (2016).

40. Aliasghari, P., Mohammadi-Ivatloo, B., Alipour, M., Abapour, M., and Zare, K. "Optimal scheduling of plug-in electric vehicles and renewable micro-grid in energy and reserve markets considering demand response program", Journal of Cleaner Production, 186, pp. 293-303 (2018).

\section{Biographies}

Parinaz Aliasghari received the BSc and MSc degrees in Electrical Engineering from University of Tabriz, Tabriz, Iran in 2011 and 2014, respectively. She is currently a Research Assistant at the Smart energy systems laboratory at the Faculty of Electrical and Computer Engineering, University of Tabriz. Her research interests include optimization, energy management, and electricity markets.

Behnam Mohammadi-Ivatloo received the BSc degree in Electrical Engineering from University of Tabriz, Tabriz, Iran in 2006, and the MSc and PhD degrees from Sharif University of Technology, Tehran, Iran in 2008, all with honors. He is currently an Associate Professor at the Faculty of Electrical and Computer Engineering, University of Tabriz, Tabriz, Iran. His main areas of research are economics, operation, and planning of intelligent energy systems in a competitive market environment.

Mehdi Abapour received the BSc and MSc degrees in Electrical Engineering from The University of Tabriz, Tabriz, Iran in 2005 and 2007, respectively, and the $\mathrm{PhD}$ degree in Electrical Engineering from The Tarbiat Modares University, Tehran, Iran in 2013. Currently, he is an Assistant Professor at the School of Electrical and Computer Engineering, University of Tabriz. His research interests include reliability, energy management, and power electronics. 